\title{
Coupled-arrangement-channel method for time-dependent wave-packet description of three-body dynamics
}

\author{
Zeki C. Kuruoğlu \\ Department of Chemistry, Bilkent University, Ankara, Turkey
}

(Received 24 May 1990)

\begin{abstract}
An alternative discretization method treating rearrangement and breakup channels on equal footing is introduced for a wave-packet description of three-body dynamics. The permutational symmetry for three identical particles is incorporated into the evolution equations of the proposed method. The method is tested on a model three-particle problem that exhibits both rearrangement and breakup channels. State-to-state $S$ matrix elements over a broad range of energies above the breakup threshold are extracted from a single wave-packet calculation.
\end{abstract}

\section{INTRODUCTION}

Time-dependent wave-packet (TDWP) methods are emerging as practical and competitive tools to study quantal scattering problems,,$^{1,2}$ and other timedependent phenomena such as photodissociation. ${ }^{3}$ There have been considerable advances in the computational implementation of the TDWP methods to elastic and inelastic collisions. ${ }^{1,4}$ However, the methodology for reactive and dissociative collisions is yet at its infancy. ${ }^{5-7}$

That the scattering process is posed as an initial-value problem entirely in the Hilbert-space setting forms the chief advantage of the time-dependent (TD) approach. In contrast, the time-independent (TI) descriptions give rise to boundary-value problems, necessitating the use of non-normalizable functions. The difficulties associated with the numerical implementation of boundary conditions for rearrangement and breakup channels are well known. The breakup channel provides an especially notorious case in this respect: Asymptotic boundary conditions are rather complicated, ${ }^{8}$ and the appropriate form to use in computations is not obvious. ${ }^{9}$ The TD approach being free of the problem of asymptotic boundary conditions would therefore be most advantageous for collisions involving rearrangement and breakup.

In a recent article, ${ }^{7}$ Kuruoğlu and Levin have demonstrated, for a three-particle model with breakup channel, that state-to-state ( sharp-energy) $S$ matrix elements for rearrangement can be extracted over a range of energies from a single wave-packet solution of the time-dependent Schrödinger equation (TDSE). The crucial factor in the success of this calculation was the expansion ansatz used to discretize the spatial degrees of freedom. In particular, the expansion basis had the flexibility to represent outgoing wave packets in all three rearrangements channels. In the present work, we explore a new expansion ansatz in which the breakup channel is represented on an equal footing with the rearrangements. The theoretical basis of this new scheme for spatial discretization is implicit in the Chandler-Gibson two-Hilbert-space theory of many-particle scattering. ${ }^{10}$

In the TDWP methods, an initial incoming wave packet representing the internal states of the separated collision partners and their free relative motion is numerically propagated in time under the full Hamiltonian. The analysis of the wave packet at asymptotic times, large enough to ensure the separation of outgoing packets in different arrangement channels, yields the $S$ matrix elements. In numerical implementation, the full Hilbert space is replaced by a finite approximation space. An approximate evolution equation on this truncated space can be formulated via a number of procedures. A nonexhaustive list includes the time-dependent variational principle, Galerkin method, and collocation method. ${ }^{11}$ The resulting system of first-order differential equations in time can be solved by a variety of integration methods. ${ }^{11,12}$ In this work, we use a propagation scheme ${ }^{13}$ based on the central-difference approximation to the time derivative. Since the real bottleneck in applications of the TDWP approach to reactive scattering lies in the space-discretization step, we will concentrate on the selection of the approximation space for threeparticle systems above the breakup threshold.

The specification of the approximation space entails, first, the selection of an appropriate set of coordinates (or momenta). In principle, the approximation space can be built from basis functions in any given set of coordinates. However, the separability of the dynamics in arrangement channels at asymptotic times cannot be exploited effectively with such a choice. As is well known from the TI theory, there is no unique set of coordinates capable of describing the four types of asymptotic separable dynamics of a three-particle system. Natural variables for separability are the Jacobi variables for rearrangement channels and hyperspherical variables for the breakup channel. If the expansion basis consists of direct-product functions in Jacobi variables of just one rearrangement, then these basis functions will be hard pressed to represent the pieces of the final wave packet emerging in other arrangement channels. To efficiently 
represent outgoing packets in different arrangement channels with a finite basis, the approximation space should be built by joining arrangement-channel subspaces, each of which ensures separability of dynamics in the respective asymptotic channel. In particular, each arrangement subspace would be spanned by a set of direct-product functions in the natural variables of that arrangement channel.

Wave packets move and spread in coordinate space. In contrast, the momentum-space wave packets retain their support. For this reason we formulate our TDWP method in momentum space, although the proposed expansion ansatz can also be used within the coordinate representation.

In our previous work, ${ }^{7}$ the approximation space was constructed from three rearrangement subspaces. Each rearrangement-channel basis consisted of (directproduct) piecewise interpolation functions in the $\mathrm{Ja}$ cobi momenta of that rearrangement. The part of the final wave packet in a given rearrangement channel could thus be described entirely within the subspace for that rearrangement, whereas the breakup part was distributed over the full approximation space. As such, this method can be considered as the time-dependent version of the pseudostate-augmented ${ }^{14}$ coupled-reactionchannel method. ${ }^{15}$ In the present work, rearrangement subspaces are more restricted, but these are augmented by a breakup subspace. In particular, the subspace for a given rearrangement $(1)(23)$ of three particles is spanned by direct products of the bound states of (23) with local interpolation functions for the relative motion of 1 . Thus, the breakup part of the final wave packet is entirely described by the breakup basis consisting of direct-product local interpolation functions in hyperspherical variables.

Note that the subspaces for two distinct arrangement channels are not orthogonal, so that the approximation space is not a simple direct sum of these subspaces. ${ }^{10}$ If each arrangement-channel basis is pushed to completeness, an overcompleteness problem would arise. In practice, with relatively small bases, the linear independence can usually be ensured. If formal or numerical linear dependences of basis functions arise, appropriate pseudoinverse techniques ${ }^{16}$ have to be employed. The nonorthogonality of the arrangement-channel subspaces, although not desirable from a computational standpoint, does not cause any formal difficulties as long as the final analysis is performed at sufficiently large times, because at such times packets emerging in different arrangement channels will be spatially separated, and, hence, orthogonal.

The proposed method is tested on a model problem involving three identical particles which interact with separable $S$-wave pair potentials. This model, having both rearrangement and breakup channels and being numerically solvable within the Faddeev formalism ${ }^{17}$ of the time-independent scattering theory, provides a nontrivial test system for the TD description of reactive and dissociative collisions.
In Sec. II, the kinematics and channel structure of the three-particle system is introduced. The description of the three-particle test problem, as well as the specification of the basis functions, is also given in this section. The expansion ansatz is introduced in Sec. III, and the long-time analysis of the wave packet is discussed in Sec. IV. The implementation of the exchange symmetry for identical particles within the present method is given in Sec. V. The computational implementation of the proposed method for the test problem is presented in Sec. VI. Here the wave-packet results are compared with results obtained within the TI Faddeev formalism. Finally, in Sec. VII we discuss the main features of the proposed method, and contrast them with other TDWP approaches.

\section{KINEMATICS, CHANNELS, AND EXPANSION BASIS}

Working in the barycentric coordinate system, the Jacobi coordinates of the rearrangement $(\alpha)(\beta \gamma)$ are denoted by $\mathbf{x}_{\alpha}$ and $\mathbf{y}_{\alpha}$, with $\mathbf{x}_{\alpha}$ being the relative coordinate of the pair $(\beta \gamma)$, and $\mathbf{y}_{\alpha}$ the relative position of the particle $\alpha$ with respect to the center of mass of the pair $(\beta \gamma)$. The canonical momenta conjugate to $\mathbf{x}_{\alpha}$ and $\mathbf{y}_{\alpha}$ are denoted by $\mathbf{p}_{\alpha}$ and $\mathbf{q}_{\alpha}$, with corresponding reduced masses being $\mu_{\alpha}$ and $M_{\alpha}$, respectively. The three-particle final states are best described by going over to hyperspherical variables: ${ }^{18}\left(\mathbf{x}_{\alpha}, \mathbf{y}_{\alpha}\right) \equiv\left(\rho, \hat{\rho}_{\alpha}\right)$, and $\left(\mathbf{p}_{\alpha}, \mathbf{q}_{\alpha}\right) \equiv\left(\kappa, \hat{\kappa}_{\alpha}\right)$. Here $\rho^{2}=2 \mu_{\alpha} x_{\alpha}^{2}+2 M_{\alpha} y_{\alpha}^{2}$, and $\kappa^{2}=p_{\alpha}^{2} /\left(2 \mu_{\alpha}\right)+q_{\alpha}^{2} /\left(2 M_{\alpha}\right)$. Although $\rho$ and $\kappa$ are common to all rearrangement channels, the set of five hyperangles $(\hat{\rho}$ or $\hat{\kappa}$ ) are dependent on $\alpha$, and can be chosen in a variety of ways. ${ }^{18}$

The kinetic-energy operator $H_{0}$ can be writen in Jacobi coordinates as $H_{0}=k_{\alpha}+K_{\alpha}$, where $k_{\alpha}=p_{\alpha}^{2} /\left(2 \mu_{\alpha}\right)$, and $K_{\alpha}=q_{\alpha}^{2} /\left(2 M_{\alpha}\right)$, with $\alpha=1,2$, or 3 . In hypershericalmomentum representation, however, $H_{0}=\kappa^{2}$. The eigenstates of $H_{0}$ are the direct-product states $\left|\mathbf{p}_{\alpha} \mathbf{q}_{\alpha}\right\rangle$. The internal Hamiltonian $h_{\alpha}$ for the pair $(\beta \gamma)$ is given as $h_{\alpha}=k_{\alpha}+V_{\alpha}$, where $V_{\alpha}$ is the interaction between particles $\beta$ and $\gamma$. Bound states of $h_{\alpha}$ are denoted $\left|\varphi_{\alpha n}\right\rangle$, with energies $\epsilon_{\alpha n}$.

The asymptotic dynamics in the rearrangement channel $\alpha$ is described by $H_{\alpha}=K_{\alpha}+h_{\alpha}$, whose eigenkets $\left|\varphi_{\alpha n} \mathbf{q}_{\alpha}\right\rangle$ are the asymptotic channel states with energies $E_{\alpha n q}=\epsilon_{\alpha n}+q_{\alpha}^{2} /\left(2 M_{\alpha}\right)$. The full Hamiltonian $H$ is then decomposed as $H=H_{0}+V$ for breakup, and $H=H_{\alpha}+V^{\alpha}$ for rearrangement channels. Here $V$ is the full interaction, and $V^{\alpha}\left(\equiv V-V_{\alpha}\right), \alpha=1,2,3$, are the channel interactions.

The basis functions for the $\alpha$-rearrangement subspace are the direct-product functions $\varphi_{\alpha n}\left(\mathbf{p}_{\alpha}\right) u_{\alpha m}\left(\mathbf{q}_{\alpha}\right), n$ $=1,2, \ldots, \mathcal{N}_{\alpha} ; m=1,2, \ldots, \mathcal{M}_{\alpha}$. Here $\left\{u_{\alpha m}\right\}$ is a suitable set of $\mathcal{M}_{\alpha}$ expansion functions for the spectator particle, and $\mathcal{N}_{\alpha}$ the number of bound states of 
the pair $\alpha$. The basis functions for the breakup channel are of the form $\varphi_{0 n}\left(\hat{\kappa}_{1}\right) u_{0 m}(\kappa), n=1,2, \cdots, \mathcal{N}_{0}$, $m=1,2, \ldots, \mathcal{M}_{0}$. Here the set $\left\{\varphi_{0 n}\left(\hat{\kappa}_{1}\right)\right\}$ discretizes the continuum of breakup channels (with fixed energy). Similarly, the set $\left\{u_{0 m}\right\}$ is the discretization basis for $\kappa$ (or energy). Although the hyperangular basis has been expressed in the variable $\hat{\kappa}_{1}$ alone, to reduce the dimension of the breakup subspace this basis could also include functions of $\hat{\kappa}_{2}$ and $\hat{\kappa}_{3}$, provided care is exercized to avoid linear dependence. The full approximation space is then the union of rearrangement and breakup subspaces. As noted in the Introduction, the subspaces for different arrangements are not orthogonal to each other, but linear dependence can be avoided, in practice, with the use of small subspaces.

Instead of giving a general discussion of how to choose the discretization bases, we will illustrate the proposed method in the context of a three-particle model. The model used consists of three identical spinless particles whose total interaction is pairwise additive, with twoparticle interactions being rank-1 separable. In particular, we have $V_{\alpha}=\left|\chi_{\alpha}\right\rangle \lambda_{\alpha}\left\langle\chi_{\alpha}\right|$, with $\chi(\mathbf{p})=\left(\beta^{2}+p^{2}\right)^{-1}$. Note that the pair potentials act only on $s$ waves and support one bound state (i.e., $\mathcal{N}_{\alpha}=1$ ). The particle masses are taken equal to proton mass $M_{p}$, and we set $M_{p}=\hbar=1$ in the rest of this article. Taking the unit of length as $\mathrm{fm}$, the resulting units for momentum, energy, and time are $\mathrm{fm}^{-1}, \mathrm{fm}^{-2}$, and $\mathrm{fm}^{2}$, respectively. We took $\beta=1.444 \mathrm{fm}^{-1}$, and $\lambda$ was chosen to give the bound-state energy of the two-nucleon system: $\epsilon=-0.053695 \mathrm{fm}^{-2}$ $(-2.226 \mathrm{MeV})$. We further restrict our attention to zero total-angular-momentum state, so that angular variables $\hat{\mathbf{p}}$ and $\hat{\mathbf{q}}$ disappear from the problem. In hyperspherical representation, the variables can be taken as $\kappa$ and $\theta_{\alpha}$, where $p_{\alpha}=\kappa \cos \theta_{\alpha}$, and $q_{\alpha}=\sqrt{4 / 3} \kappa \sin \theta_{\alpha}$.

The expansion bases in the variables $q_{\alpha}, \theta_{\alpha}$, and $\kappa$ are taken as piecewise interpolation functions ${ }^{11}$ (quadratic polynomials in this work). For this purpose, cutoff values $q_{\alpha, \max }$ and $\kappa_{\max }$ are introduced by considering the momentum-space support of the wave packet. For a given variable $x(=q, \kappa$, or $\theta)$, the interval $\left[0, x_{\max }\right]$ is partitioned into $\mathcal{I}_{x}$ subintervals, and a set of $2 \mathcal{I}_{x}-3$ quadratic local-interpolation functions $u_{i}(x)$ is defined on this mesh. ${ }^{11}$ The partition meshes do not have to be evenly distributed, but are chosen to have a higher density in regions where the wave packet is expected to have appreciable amplitude. If the set $\left\{x_{i}\right\}$ stands for the ordered collection of endpoints and midpoints of subintervals (with 0 and $x_{\max }$ excluded), then the interpolation functions have the property ${ }^{11} u_{j}\left(x_{i}\right)=\delta_{i j}$, $i, j=1,2, \ldots, 2 \mathcal{I}_{x}-3$. The dimensions of the rearrangement subspaces are $\mathcal{N}_{\alpha} \mathcal{M}_{\alpha}$, where $\mathcal{M}_{\alpha}=2 \mathcal{I}_{q_{\alpha}}-3$. Using $\mathcal{N}_{0 \alpha}\left(=2 \mathcal{I}_{\theta_{\alpha}}-3\right)$ interpolation functions $\varphi_{0 n}\left(\theta_{\alpha}\right)$ for each $\theta_{\alpha}, \alpha=1,2,3$, and $\mathcal{M}_{0}\left(=2 \mathcal{I}_{\kappa}-3\right)$ functions for $\kappa$, the dimension of the breakup subspace is $\mathcal{N}_{0} \mathcal{M}_{0}$, where $\mathcal{N}_{0}=\mathcal{N}_{01}+\mathcal{N}_{02}+\mathcal{N}_{03}$. The set of $\mathcal{N}_{0}$ interpolation functions in $\theta_{1}, \theta_{2}$, and $\theta_{3}$ will collectively be denoted by $\varphi_{0 n}$, $n=1,2, \ldots, \mathcal{N}_{0}$.

\section{EXPANSION ANSATZ FOR THE WAVE PACKET}

Let us consider an initial incoming wave packet corresponding to a collision in which particle $\alpha$ is incident on a bound state $\varphi_{\alpha n_{0}}$ of the pair $(\beta \gamma)$ :

$$
\left|\Phi_{\alpha n_{0} q_{0}}(0)\right\rangle=\left|\varphi_{\alpha n_{0}}\right\rangle\left|f_{\alpha q_{0}}\right\rangle
$$

where $f_{\alpha q_{0}}\left(q_{\alpha}\right)$ is an incoming wave packet for the relative motion of particle $\alpha$ with average momentum $q_{0}$, and average position $y_{0}$. We take $y_{0}$ to be well outside the range of $V^{\alpha}$. The form of $f_{\alpha q_{0}}$ is

$$
f_{\alpha q_{0}}\left(q_{\alpha}\right)=A \exp \left[-\left(q_{\alpha}-q_{0}\right)^{2} w^{2} / 2\right] \exp \left[i y_{0}\left(q_{\alpha}-q_{0}\right)\right]
$$

where $A$ is a normalization constant and $w$ is the width parameter. The free time evolution under $H_{\alpha}$ of the initial wave packet is given simply as $\left|\Phi_{\alpha n_{0} q_{0}}(t)\right\rangle$ $=\left|\varphi_{\alpha n_{0}}(t)\right\rangle\left|f_{\alpha q_{0}}(t)\right\rangle$, with

$$
\begin{aligned}
& \left\langle p_{\alpha} \mid \varphi_{\alpha n_{0}}(t)\right\rangle=\exp \left(-i \epsilon_{\alpha n_{0}} t\right) \varphi_{\alpha n_{0}}\left(p_{\alpha}\right) \\
& \left\langle q_{\alpha} \mid f_{\alpha q_{0}}(t)\right\rangle=\exp \left[-i q_{\alpha}^{2} t /\left(2 M_{\alpha}\right)\right] f_{\alpha q_{0}}\left(q_{\alpha}\right) .
\end{aligned}
$$

Note that average momentum and momentum dispersion $\Delta q$ of the free wave packet $|f\rangle$ do not change with time. That is, the support (or, envelope) of the momentum distribution remains unchanged, and time evolution manifests itself as increased oscillations. Actually, this feature is true of not just free wave packets, but also of packets evolving under a potential, and should be contrasted with the moving and spreading of wave packets in coordinate space.

The solution $\left|\Psi_{\alpha n_{0} q_{0}}(t)\right\rangle$ of the time-dependent Schrödinger equation, subject to the initial condition $\left|\Psi_{\alpha n_{0} q_{0}}(0)\right\rangle=\left|\Phi_{\alpha n_{0} q_{0}}(0)\right\rangle$ is written as the sum of four arrangement-channel components:

$$
\left|\Psi_{\alpha n_{0} q_{0}}(t)\right\rangle=\sum_{\beta=0}^{3}\left|\Psi_{\alpha n_{0} q_{0}}^{(\beta)}(t)\right\rangle
$$

with the initial condition now reading

$$
\left|\Psi_{\alpha n_{0} q_{0}}^{(\beta)}(0)\right\rangle=\delta_{\alpha \beta}\left|\Phi_{\alpha n_{0} q_{0}}(0)\right\rangle, \quad \beta=0,1,2,3 .
$$

Each component $\left|\Psi^{(\beta)}\right\rangle$ is now expanded as

$$
\left|\Psi_{\alpha n_{0} q_{0}}^{(\beta)}\right\rangle=\sum_{n=1}^{\mathcal{N}_{\beta}} \sum_{m=1}^{\mathcal{M}_{\beta}}\left|\varphi_{\beta n} u_{\beta m}\right\rangle c_{\beta n m, \alpha n_{0} q_{0}}(t) .
$$

The initial condition for expansion coefficients becomes

$$
c_{\beta n m, \alpha n_{0} q_{0}}(0)=\delta_{\beta \alpha} \delta_{n n_{0}} a_{\alpha m}(0)
$$

where $a_{\alpha m}(0)$ are the expansion coefficients for $f_{\alpha q_{0}}$ in the basis $\left\{u_{\alpha m}\right\}$, viz.,

$$
f_{\alpha q_{0}}\left(q_{\alpha}\right)=\sum_{m=1}^{\mathcal{M}_{\alpha}} u_{\alpha m}\left(q_{\alpha}\right) a_{\alpha m}(0) .
$$


Substituting Eqs. (5) and (7) into the TDSE, and projecting with basis functions $\left|\varphi_{\gamma n^{\prime}} u_{\gamma m^{\prime}}\right\rangle$, we obtain a set of first-order differential equations for the expansion coefficients:

$$
\begin{aligned}
& \sum_{\beta=0}^{3} \sum_{n=1}^{\mathcal{N}_{\beta}} \sum_{m=1}^{\mathcal{M}_{\beta}}\left\langle\varphi_{\gamma n^{\prime}} u_{\gamma m^{\prime}}|H| \varphi_{\beta n} u_{\beta m}\right\rangle c_{\beta n m}(t) \\
& =i \sum_{\beta=0}^{3} \sum_{n=1}^{\mathcal{N}_{\beta}} \sum_{m=1}^{\mathcal{M}_{\beta}}\left\langle\varphi_{\gamma n^{\prime}} u_{\beta m^{\prime}} \mid \varphi_{\beta n} u_{\beta m}\right\rangle \dot{c}_{\beta n m}(t)
\end{aligned}
$$

where $\gamma=0,1,2,3, n^{\prime}=1, \ldots, \mathcal{N}_{\gamma}$, and $m^{\prime}=1, \ldots, \mathcal{M}_{\gamma}$. Here the initial-state labels $\left(\alpha n_{0} q_{0}\right)$ have been suppressed. Collecting the coefficients $c_{\beta n m}$ in the column vector c, the matrix elements $\left\langle\varphi_{\gamma n^{\prime}} u_{\gamma m^{\prime}}|H| \varphi_{\beta n} u_{\beta m}\right\rangle$ in the matrix $\mathbf{H}$, and the overlap matrix elements $\left\langle\varphi_{\gamma n^{\prime}} u_{\gamma m^{\prime}} \mid \varphi_{\beta n} u_{\beta m}\right\rangle$ in the nonorthogonality matrix $\Delta$, Eq. (10) reads

$$
i \ddot{\mathbf{c}}(t)=\Delta^{-1} \mathbf{H} \mathbf{c}(t) .
$$

If $\boldsymbol{\Delta}$ is singular formally or numerically, $\boldsymbol{\Delta}^{-1}$ is to be understood as the pseudo-inverse. ${ }^{16}$

\section{WAVE-PACKET PROPAGATION AND ASYMPTOTIC ANALYSIS}

To solve Eq. (11), we use a step-by-step propagation scheme based on the central-difference approximation to the time derivative. Denoting the time step with $\delta t$, the propagation procedure reads

$$
\mathbf{c}\left(t_{k+1}\right)=\mathbf{c}\left(t_{k-1}\right)-2 i \delta t \boldsymbol{\Delta}^{-1} \mathbf{H} \mathbf{c}\left(t_{k}\right) .
$$

where $t_{k}=k \delta t$. To start the propagation, we need $\mathbf{c}\left(t_{0}=0\right)$, and $\mathbf{c}\left(t_{1}=\delta t\right)$. Equation (8) determines $\mathbf{c}(0)$, and $\mathbf{c}(\delta t)$ can be obtained, e.g., by a forward-difference approximation of Eq. (11).

Denoting by $t_{\min }$ the minimum time of propagation needed for the emergence of the wave packet from the interaction region, the probability amplitude for the system to be in state $\left|\varphi_{\beta n^{\prime}}(T) q_{\beta}^{\prime}\right\rangle$ for $\beta=1,2,3$, at a sampling time $T\left(\geq t_{\min }\right)$ is given as

$$
\begin{aligned}
\left\langle\varphi_{\beta n^{\prime}}(T) q_{\beta}^{\prime}\right| & \left.\Psi_{\alpha n_{0} q_{0}}(T)\right\rangle \\
& =\left\langle\varphi_{\beta n^{\prime}}(T) q_{\beta}^{\prime}\left|S_{\beta \alpha}\right| \Phi_{\alpha n_{0} q_{0}}(T)\right\rangle,
\end{aligned}
$$

where $S_{\beta \alpha}$ is the rearrangement scattering operator for the $\alpha \rightarrow \beta$ transitions. To obtain the state-to-state $S$ matrix elements, we invoke the energy-conserving property of the $S$ operator. ${ }^{19}$ For the present model of $s$-wave interactions and zero total-angular-momentum states, we have

$\left\langle\varphi_{\beta n^{\prime}} q_{\beta}^{\prime}\left|S_{\beta \alpha}\right| \varphi_{\alpha n} q_{\alpha}\right\rangle$

$$
=\frac{\delta\left(E_{\beta n^{\prime} q^{\prime}}-E_{\alpha n q}\right)}{\sqrt{M_{\beta} M_{\alpha} q_{\beta}^{\prime} q_{\alpha}}} \hat{S}_{\beta n^{\prime}, \alpha n}\left(E_{\alpha n q}\right)
$$

where $\hat{S}_{\beta n^{\prime}, \alpha n}$ is the reduced $S$ matrix whose absolutevalue square gives the probability for the transition $\left(\alpha n \rightarrow \beta n^{\prime}\right)$. Use of Eq. (14) in Eq. (13) gives

$$
\hat{S}_{\beta n^{\prime}, \alpha n_{0}}\left(E_{\beta n^{\prime} q^{\prime}}\right)=N_{\beta \alpha} \frac{\left\langle\varphi_{\beta n^{\prime}}(T) q_{\beta}^{\prime} \mid \Psi_{\alpha n_{0} q_{0}}(T)\right\rangle}{\left\langle q_{\alpha} \mid f_{\alpha q_{0}}(T)\right\rangle}
$$

where $q_{\alpha}$ is determined from $E_{\beta n^{\prime} q^{\prime}}=E_{\alpha n_{0} q}$, and $N_{\beta \alpha}$ $=\sqrt{M_{\beta} q_{\beta}^{\prime} /\left(M_{\alpha} q_{\alpha}\right)}$.

calculations the conservation of energy (in a state-to-state sense ${ }^{20}$ ) will be satisfied only approximately, the $S$-matrix elements extracted via Eq. (15) will exhibit a dependence on the sampling time $T$. The stability of the $S$ matrix with respect to $T$ is a measure of the adequacy of the computational parameters. Also the sampling time cannot be taken to be arbitrarily large. A given finite expansion basis in momentum space implies a finite coordinate-space domain which is determined by the coordinate-space support of the basis functions. (For momentum-space interpolation functions defined on a momentum mesh, the finer the momentum discretization, the larger will be the corresponding coordinate-space support.) Hence, there is a maximum time $t_{\max }$ of meaningful propagation after which the wave packet starts to reflect from the boundaries of the implicit coordinate-space domain. Therefore the momentum-space discretization basis should be large enough to ensure a time period of free propagation between $t_{\min }$ and $t_{\max }$, during which the $S$ matrix can be extracted. By periodically constructing the coordinatespace image of the wave packet, and computing its average position and position dispersion, the appropriate time interval $t_{\min } \leq T \leq t_{\max }$ that ensures product separation and reflection-free time evolution can be ascertained.

Another technical point is that the space and time discretizations produce numerical scattering even for the free wave packet, especially for large propagation times. To cancel these spurious effects in Eq.(15), it is imperative to also treat numerically the time evolution of free wave packets. In other words, since the $S$ operators basically compare the $H$ dynamics with the $H_{\alpha}$ dynamics, a channel Hamiltonian $H_{\alpha}$, whether it occurs in the context of the time evolution of $\Psi$ under $H$, or in relation to free time evolution of $\left|\varphi_{\alpha} f_{\alpha}\right\rangle$ under $H_{\alpha}$, should be treated at the same level of approximation. Therefore, we use in Eq. (15), not the analytical form of the state $f_{\alpha q_{0}}(T)$ as given in Eq. (4), but the numerical one, which is generated employing the same expansion basis and time-propagation scheme as for the full dynamics. ${ }^{21}$

\section{THREE IDENTICAL PARTICLES}

Let $\left|\Psi_{S n_{0} q_{0}}(t)\right\rangle$ be the solution of the TDSE for three identical spinless bosons subject to the symmetrized initial condition ${ }^{22}$ 


$$
\begin{aligned}
\left|\Psi_{S n_{0} q_{0}}(0)\right\rangle=\frac{1}{\sqrt{3}}[ & \left.\Phi_{1 n_{0} q_{0}}(0)\right\rangle+\left|\Phi_{2 n_{0} q_{0}}(0)\right\rangle \\
+ & \left.\left|\Phi_{3 n_{0} q_{0}}(0)\right\rangle\right],
\end{aligned}
$$

where initial wave packets $\left|\Phi_{\alpha n_{0} q_{0}}(0)\right\rangle, \alpha=1,2,3$ are chosen to be symmetric under the permutation $P_{\beta \gamma}$. Denoting the cyclic permutations of three particles with $P_{123}$ and $P_{132}$, and using the permutation properties $P_{123}\left|\Phi_{1}(t)\right\rangle=\left|\Phi_{2}(t)\right\rangle$ and $P_{132}\left|\Phi_{1}(t)\right\rangle=\left|\Phi_{3}(t)\right\rangle$, we have $\left|\Psi_{S}(0)\right\rangle=1 / \sqrt{3}\left(I+P_{123}+P_{132}\right)\left|\Phi_{1}(0)\right\rangle$. Here we suppressed the initial-state labels $\left(n_{0} q_{0}\right)$. Provided the approximations used to obtain $\left|\Psi_{S}(t)\right\rangle$ from the symmetrized initial wave packet $\left|\Psi_{S}(0)\right\rangle$ treat all particles identically, $\left|\Psi_{S}(t)\right\rangle$ will remain symmetrized, and we can write

$$
\left|\Psi_{S n_{0} q_{0}}(t)\right\rangle=\frac{1}{\sqrt{3}}\left(I+P_{123}+P_{132}\right)\left|\Psi_{1 n_{0} q_{0}}(t)\right\rangle .
$$

The projection of the wave packet (at asymptotic times) onto the symmetrized channel state $\left|\varphi_{n} q^{\prime}\right\rangle_{S}$ $\left[\equiv 1 / \sqrt{3}\left(I+P_{123}+P_{132}\right)\left|\varphi_{1 n^{\prime}} q_{1}^{\prime}\right\rangle\right]$ gives the probability amplitude for observing a particle with relative momentum $q^{\prime}$ while the remaining pair is in the bound state $n^{\prime}$ :

$$
\begin{aligned}
s\left\langle\varphi_{n^{\prime}}\right. & (T) q^{\prime}\left|\Psi_{S n_{0} q_{0}}(T)\right\rangle \\
& =\sqrt{3}\left\langle\varphi_{1 n^{\prime}}(T) q_{1}^{\prime} \mid \Psi_{S n_{0} q_{0}}\right\rangle, \\
& =\left\langle\varphi_{1 n^{\prime}}(T) q_{1}^{\prime}\left|\left(I+P_{123}+P_{132}\right)\right| \Psi_{1 n_{0} q_{0}}\right\rangle, \\
& \equiv\left\langle\varphi_{1 n^{\prime}}(T) q_{1}^{\prime}\left|\mathcal{S}_{n^{\prime} n_{0}}\right| \Phi_{1 n_{0} q_{0}}(T)\right\rangle,
\end{aligned}
$$

where we used Eq. (17) and $\left(I+P_{123}+P_{132}\right)^{2}$ $=3\left(I+P_{123}+P_{132}\right)$ to obtain Eq. (18), and introduced the (physical) symmetrized rearrangement $S$ operator $\mathcal{S}_{n^{\prime} n_{0}}$. Using the energy-conserving property of the scattering operator, the identical-particle version of Eq. (15) comes out as

$$
\begin{aligned}
\hat{\mathcal{S}}_{n^{\prime} n_{0}}\left(E_{1 n^{\prime} q^{\prime}}\right) & =\sqrt{3} N \frac{\left\langle\varphi_{1 n^{\prime}}(T) q_{1}^{\prime} \mid \Psi_{S n_{0} q_{0}}\right\rangle}{\left\langle q_{1} \mid f_{1 q_{0}}(T)\right\rangle} \\
& =N \frac{\left\langle\varphi_{1 n^{\prime}}(T) q_{1}^{\prime}\left|\left(I+P_{123}+P_{132}\right)\right| \Psi_{1 n_{0} q_{0}}(T)\right\rangle}{\left\langle q_{1} \mid f_{1 q_{0}}(T)\right\rangle}
\end{aligned}
$$

where $N=\sqrt{q_{1}^{\prime} / q_{1}}$, and $q_{1}$ is determined from $E_{1 n^{\prime} q^{\prime}}$ $=E_{1 n_{0} q}$, i.e.,

$$
\epsilon_{1 n^{\prime}}+\frac{3}{4} q_{1}^{\prime 2}=\epsilon_{1 n_{0}}+\frac{3}{4} q_{1}^{2} .
$$

Note that $\hat{\mathcal{S}}_{n^{\prime} n}$ can be expressed in two equivalent forms in terms of distinguishable-particle $S$ matrix elements:

$$
\begin{aligned}
\hat{\mathcal{S}}_{n^{\prime} n} & =\hat{S}_{1 n^{\prime}, 1 n}+\hat{S}_{1 n^{\prime}, 2 n}+\hat{S}_{1 n^{\prime}, 3 n}, \\
& =\hat{S}_{1 n^{\prime}, 1 n}+\hat{S}_{2 n^{\prime}, 1 n}+\hat{S}_{3 n^{\prime}, 1 n},
\end{aligned}
$$

which follow from Eqs. (15) and (22) using permutation properties such as $P_{123}\left|\Psi_{1 n_{0} q_{0}}\right\rangle=\left|\Psi_{2 n_{0} q_{0}}\right\rangle$, and $P_{123}\left|\varphi_{1 n^{\prime}} q_{1}^{\prime}\right\rangle=\left|\varphi_{2 n^{\prime}} q_{2}^{\prime}\right\rangle$. Hence, the symmetrized $S$ matrix can be obtained from Eq. (22) by solving Eq. (11) once for $\left|\Psi_{1 n_{0} q_{0}}\right\rangle$ as if the particles were distinguishable.

However, the dimension of the matrix problem can be reduced by block diagonalizing Eq. (11) according to the irreducible representations of the permutation group $S_{3}$, and only the totally symmetric block has to be solved. That is, we do not have to work within the full approximation space, but only within the symmetric subspace. Towards this end, we first rewrite Eq. (5) as

$$
\left|\Psi_{\alpha n_{0} q_{0}}\right\rangle=\sum_{\beta=1}^{3}\left(\left|\Psi_{\alpha n_{0} q_{0}}^{(\beta)}\right\rangle+\left|\Psi_{\alpha n_{0} q_{0}}^{(0 \beta)}\right\rangle\right), \alpha=1,2,3,
$$

where we split the breakup components $\left|\Psi^{(0)}\right\rangle$ into three subcomponents $\left|\Psi^{(0 \beta)}\right\rangle, \beta=1,2,3$, corresponding to three different choices of $\theta_{\beta}$. From permutation symmetry of the problem, we have $\left|\Psi_{1}^{(1)}\right\rangle=P_{132}\left|\Psi_{2}^{(2)}\right\rangle$ $=P_{123}\left|\Psi_{3}^{(3)}\right\rangle,\left|\Psi_{1}^{(2)}\right\rangle=P_{123}\left|\Psi_{3}^{(1)}\right\rangle=P_{132}\left|\Psi_{2}^{(3)}\right\rangle$, and $\left|\Psi_{1}^{(3)}\right\rangle=P_{123}\left|\Psi_{3}^{(2)}\right\rangle=P_{132}\left|\Psi_{2}^{(1)}\right\rangle$. The breakup subcomponents $\left|\Psi_{\alpha}^{(0 \beta)}\right\rangle$ transform under cyclic permutations just like the rearrangement components $\left|\Psi_{\alpha}^{(\beta)}\right\rangle$. As a result, $\left|\Psi_{S n_{0} q_{0}}\right\rangle$ can be written as

$$
\left|\Psi_{S n_{0} q_{0}}\right\rangle=\frac{1}{\sqrt{3}}\left(I+P_{123}+P_{132}\right)\left(\left|\Psi_{S n_{0} q_{0}}^{(1)}\right\rangle+\left|\Psi_{S n_{0} q_{0}}^{(01)}\right\rangle\right),
$$

where

$$
\left|\Psi_{S n_{0} q_{0}}^{(1)}\right\rangle=\sum_{\alpha=1}^{3}\left|\Psi_{\alpha n_{0} q_{0}}^{(1)}\right\rangle, \quad\left|\Psi_{S n_{0} q_{0}}^{(01)}\right\rangle=\sum_{\alpha=1}^{3}\left|\Psi_{\alpha n_{0} q_{0}}^{(01)}\right\rangle .
$$

The symmetrized initial condition (16) now becomes $\left|\Psi_{S n_{0} q_{0}}^{(1)}(0)\right\rangle=\left|\Phi_{1 n_{0} q_{0}}(0)\right\rangle$, and $\left|\Psi_{S n_{0} q_{0}}^{(01)}(0)\right\rangle=0$.

The totally symmetric subspace of the full approximation space is spanned by the union of the sets $\left\{\left(I+P_{123}+P_{132}\right)\left|\varphi_{1 n} u_{1 m}\right\rangle, n=1, \ldots, \mathcal{N}_{1}, m=\right.$ $\left.1, \ldots, \mathcal{M}_{1}\right\}$, and $\left\{\left(I+P_{123}+P_{132}\right)\left|\varphi_{0 n} u_{0 m}\right\rangle, n=\right.$ $\left.1,2, \ldots, \mathcal{N}_{01}, m=1,2, \ldots, \mathcal{M}_{0}\right\}$. We now expand the symmetrized rearrangement and breakup components as 


$$
\begin{aligned}
\left|\Psi_{S n_{0} q_{0}}^{(1)}(t)\right\rangle & =\sum_{n=1}^{\mathcal{N}_{1}} \sum_{m=1}^{\mathcal{M}_{1}}\left|\varphi_{1 n} u_{1 m}\right\rangle c_{1 n m}^{S}, \\
\left|\Psi_{S n_{0} q_{0}}^{(01)}(t)\right\rangle & =\sum_{n=1}^{\mathcal{N}_{01}} \sum_{m=1}^{\mathcal{M}_{0}}\left|\varphi_{0 n} u_{0 m}\right\rangle c_{0 n m}^{S}(t) .
\end{aligned}
$$

with the initial conditions $c_{1 n m}^{S}(0)=\delta_{n n_{0}} a_{1 m}(0)$, and $c_{0 n m}^{S}(0)=0$. Here $a_{1 m}$ are the expansion coefficients of $f_{1 q_{0}}$ in the basis $\left\{u_{1 m}\right\}$. Substituting (29) and (30) into the TDSE, and taking inner products with $\left|\varphi_{1 n^{\prime}} u_{1 m^{\prime}}\right\rangle$ and $\left|\varphi_{0 n^{\prime}} u_{0 m^{\prime}}\right\rangle$ in turn, we obtain the symmetrized version of Eq. (11) as

$$
\left(\begin{array}{ll}
\mathbf{H}_{11}^{\mathbf{S}} & \mathbf{H}_{10}^{\mathbf{S}} \\
\mathbf{H}_{01}^{\mathbf{S}} & \mathbf{H}_{00}^{\mathbf{S}}
\end{array}\right)\left(\begin{array}{c}
\mathbf{c}_{1}^{\mathbf{S}} \\
\mathbf{c}_{0}^{\mathbf{S}}
\end{array}\right)=i\left(\begin{array}{cc}
\Delta_{11}^{\mathbf{S}} & \Delta_{10}^{\mathbf{S}} \\
\boldsymbol{\Delta}_{01}^{\mathbf{S}} & \Delta_{00}^{\mathbf{S}}
\end{array}\right)\left(\begin{array}{c}
\dot{\mathbf{c}}_{1}^{\mathbf{S}} \\
\dot{\mathbf{c}}_{0}^{\mathbf{S}}
\end{array}\right) \text {. }
$$

Here we introduced the matrix notation

$$
\begin{aligned}
& \mathbf{A}_{\mathbf{i}^{\prime} \mathbf{i}}^{\mathbf{S}}=\left\{\left\langle\varphi_{i^{\prime} n^{\prime}} u_{i^{\prime} m^{\prime}}\left|A\left(I+P_{123}+P_{132}\right)\right| \varphi_{i n} u_{i m}\right\rangle\right\} \\
& \mathbf{c}_{\mathbf{i}}^{\mathbf{s}}=\operatorname{col}\left\{c_{i n m}^{S}\right\},
\end{aligned}
$$

with $A=H$, or $I, i=0,1$, and $i^{\prime}=0,1$.

\section{COMPUTATIONAL IMPLEMENTATION AND RESULTS}

Since the two-particle subsystems in our model support just one bound state each, the bound-state indices are suppressed in this section. In particular, the symmetrized rearrangement $S$ matrix elements $\mathcal{S}_{n^{\prime} n}\left(E_{1 n q}\right)$, $n=n^{\prime}=1$, are simply referred to as the elastic $S$ matrix, and are denoted by $\mathcal{S}_{\text {el }}\left(E_{1 q}\right)$, where $E_{1 q}=\epsilon+3 q^{2} / 4$. As is well known, ${ }^{17}$ the three-particle problem with separable $S$-wave pair potentials can be solved to arbitrary numerical precision using the momentum-space Faddeev integral equations. The results labeled as exact in Tables I and II were obtained by solving the Faddeev equations ${ }^{17}$ for the transition operators with a Schwinger-type variational method, ${ }^{23}$ and are accurate to three significant figures.

For the calculations reported in this article, the parameters of the initial wave packet were taken as $q_{0}=4.0$ $\mathrm{fm}^{-1}, y_{0}=9.0 \mathrm{fm}$, and $w=2.0 \mathrm{fm}$. The momentum probability density of the initial wave packet is appreciable (greater than 0.01 ) in the range $3.0 \leq q \leq 5.0$. The computational domain in momentum space was restricted by the cutoff values $q_{\max }=6.4 \mathrm{fm}^{-1}$, and $\kappa_{\max }=6.0 \mathrm{fm}^{-1}$. The interval $\left[0, q_{\max }\right]$ for $q_{1}$ was divided into 30 finite elements, giving rise to 59 quadratic interpolation functions. A denser set of mesh points (with a spacing of 0.1 ) was used in the interval from 3.2 to 4.8 where the initial wave packet has most of its amplitude. Similarly, the division of the interval $\left[0, \kappa_{\max }\right]$ into 21 finite elements gives 41 quadratic interpolation functions for $\kappa$. Again, 16 of the finite elements cover the subinterval [2.1,4.5], which roughly corresponds to the energy support of the initial wave packet. Finally, the interval $[0, \pi / 2]$ for $\theta_{1}$ was divided into 7 equal finite elements, resulting in 13 basis functions. Thus, with $\mathcal{M}_{1}=59, \mathcal{M}_{0}=41$, and $\mathcal{N}_{01}=13$, the dimensions of the $\mathbf{H}$ and $\boldsymbol{\Delta}$ matrices in the present set of calculations were 592 .

The value $\delta t=0.002$ was used in the step-by-step propagation scheme. With the system of units adopted, the time unit is $\mathrm{fm}^{2}$, which, however, is supressed in the rest of the article. The norm of the wave packet was conserved to better than 0.001 . The evolution of the wave packet was monitored by periodically calculating its coordinatespace image in order to guarantee that at the sampling times the wave packet is in the asymptotic region and free of boundary reflection.

The elastic part $\left|\Psi_{\mathrm{el}}\right\rangle$ of the final wave packet (i.e., the part that corresponds to a spectator particle moving away from the bound pair) will have the form

$$
\left|\Psi_{\mathrm{el}}(t)\right\rangle=\frac{1}{\sqrt{3}}\left(I+P_{123}+P_{132}\right)\left|\varphi_{1}(t) g_{1 q_{0}}(t)\right\rangle
$$

TABLE I. $\left\langle y_{1}\right\rangle_{\text {free }},\left\langle y_{1}\right\rangle_{\mathrm{el}}, \Delta y_{\mathrm{el}}$, and $\left\langle\mathcal{S}_{\mathrm{el}}\right\rangle$ as a function of the sampling time $T$. The computational parameters are $q_{0}=4.0 \mathrm{fm}^{-1}, y_{0}=9.0 \mathrm{fm}, w=2.0 \mathrm{fm}, q_{\max }=6.4 \mathrm{fm}^{-1}, \kappa_{\max }=6.0 \mathrm{fm}^{-1}, \mathcal{M}_{1}=59$, $\mathcal{M}_{0}=41, \mathcal{N}_{01}=13$, and $\delta t=0.002$.

\begin{tabular}{cccccc}
\hline \hline$T$ & $\left\langle y_{1}\right\rangle_{\text {free }}$ & $\left\langle y_{1}\right\rangle_{\text {el }}$ & $\Delta y_{\text {el }}$ & $\operatorname{Re}\left\langle\mathcal{S}_{\text {el }}\right\rangle$ & $\operatorname{Im}\left\langle\mathcal{S}_{\text {el }}\right\rangle$ \\
\hline 2.50 & 6.01 & 6.16 & 1.93 & 0.955 & 0.139 \\
2.75 & 7.51 & 7.67 & 2.03 & 0.955 & 0.138 \\
3.00 & 9.01 & 9.18 & 2.15 & 0.955 & 0.138 \\
3.25 & 10.51 & 10.68 & 2.28 & 0.955 & 0.137 \\
3.50 & 12.01 & 12.19 & 2.43 & 0.955 & 0.137 \\
3.75 & 13.52 & 13.70 & 2.60 & 0.955 & 0.137 \\
4.00 & 15.03 & 15.21 & 2.80 & 0.955 & 0.136 \\
4.50 & 18.06 & 18.24 & 3.27 & 0.954 & 0.136 \\
5.00 & 21.09 & 21.28 & 3.84 & 0.954 & 0.136 \\
5.50 & 24.14 & 24.33 & 4.56 & 0.954 & 0.136 \\
6.00 & 27.19 & 27.38 & 5.41 & 0.955 & 0.136 \\
Exact & & & & 0.953 & 0.145 \\
\hline \hline
\end{tabular}


TABLE II. Exact and wave-packet results for the symmetrized rearrangement of the $S$ matrix $\hat{\mathcal{S}}_{\mathrm{el}}\left(E_{1 q}\right)$ for a range of energies. $E_{1 q}=\epsilon+3 q^{2} / 4$. Computational parameters are the same as in Table I.

\begin{tabular}{|c|c|c|c|c|c|c|}
\hline \multirow[b]{2}{*}{$q$} & & \multirow[b]{2}{*}{$\hat{\mathcal{S}}_{\mathrm{el}}^{\text {exact }}\left(E_{1 q}\right)$} & \multicolumn{4}{|c|}{$\hat{\mathcal{S}}_{\mathrm{el}}\left(E_{1 q}\right)$ extracted from wave packet at } \\
\hline & & & $T=2.5$ & $T=3.0$ & $T=3.5$ & $T=4.0$ \\
\hline \multirow[t]{2}{*}{3.0} & $\operatorname{Re}$ & 0.836 & 0.821 & 0.863 & 0.868 & 0.865 \\
\hline & $\operatorname{Im}$ & 0.296 & 0.292 & 0.285 & 0.275 & 0.259 \\
\hline \multirow[t]{2}{*}{3.2} & $\operatorname{Re}$ & 0.875 & 0.875 & 0.885 & 0.880 & 0.864 \\
\hline & $\operatorname{Im}$ & 0.256 & 0.241 & 0.247 & 0.243 & 0.241 \\
\hline \multirow[t]{2}{*}{3.4} & $\operatorname{Re}$ & 0.908 & 0.910 & 0.912 & 0.912 & 0.911 \\
\hline & $\operatorname{Im}$ & 0.220 & 0.206 & 0.211 & 0.210 & 0.207 \\
\hline \multirow[t]{2}{*}{3.6} & $\mathrm{Re}$ & 0.928 & 0.936 & 0.933 & 0.932 & 0.930 \\
\hline & $\operatorname{Im}$ & 0.190 & 0.181 & 0.182 & 0.180 & 0.179 \\
\hline \multirow[t]{2}{*}{3.8} & $\operatorname{Re}$ & 0.945 & 0.950 & 0.948 & 0.948 & 0.947 \\
\hline & $\operatorname{Im}$ & 0.163 & 0.162 & 0.160 & 0.158 & 0.158 \\
\hline \multirow[t]{2}{*}{4.0} & $\operatorname{Re}$ & 0.958 & 0.960 & 0.960 & 0.960 & 0.960 \\
\hline & Im & 0.140 & 0.137 & 0.134 & 0.134 & 0.133 \\
\hline \multirow[t]{2}{*}{4.2} & $\operatorname{Re}$ & 0.966 & 0.968 & 0.970 & 0.970 & 0.971 \\
\hline & $\operatorname{Im}$ & 0.122 & 0.115 & 0.113 & 0.112 & 0.112 \\
\hline \multirow[t]{2}{*}{4.4} & $\mathrm{Re}$ & 0.977 & 0.977 & 0.977 & 0.977 & 0.977 \\
\hline & $\operatorname{Im}$ & 0.103 & 0.0947 & 0.0950 & 0.0942 & 0.0941 \\
\hline \multirow[t]{2}{*}{4.6} & $\operatorname{Re}$ & 0.983 & 0.983 & 0.982 & 0.983 & 0.984 \\
\hline & $\operatorname{Im}$ & 0.0888 & 0.0826 & 0.0803 & 0.0790 & 0.0789 \\
\hline \multirow[t]{2}{*}{4.8} & $\operatorname{Re}$ & 0.986 & 0.980 & 0.968 & 0.950 & 0.931 \\
\hline & $\operatorname{Im}$ & 0.0762 & 0.0643 & 0.0680 & 0.0741 & 0.0781 \\
\hline \multirow[t]{2}{*}{5.0} & $\operatorname{Re}$ & 0.989 & 0.987 & 0.976 & 0.980 & 0.968 \\
\hline & Im & 0.0653 & 0.0716 & 0.0796 & 0.0750 & 0.0750 \\
\hline
\end{tabular}

where $g_{1 q_{0}}\left(q_{1}, t\right)=\sqrt{3}\left\langle\varphi_{1}(t) q_{1} \mid \Psi_{S q_{0}}(t)\right\rangle$. Note that Eq. (21) implies, for aymptotic times $T$,

$$
g_{1 q_{0}}(q, T)=\hat{\mathcal{S}}_{\mathrm{el}}\left(E_{1 q}\right) f_{1 q_{0}}(q, T) .
$$

On the other hand, the piece representing breakup will be $\left|\Psi_{\text {bp }}\right\rangle \equiv\left|\Psi_{S}\right\rangle-\left|\Psi_{\text {el }}\right\rangle$.

Since $\left|\hat{\mathcal{S}}_{\text {el }}\right| \leq 1$, the momentum support of the spectator packet $g_{1 q_{0}}(T)$ is basically that of the free packet $f_{1 q_{0}}(T)$. Also, the total elastic probability is given as $\left\langle\Psi_{\mathrm{el}}(T) \mid \Psi_{\mathrm{el}}(T)\right\rangle=\left\langle g_{1 q_{0}}(T) \mid g_{1 q_{0}}(T)\right\rangle$, where we used the asymptotic orthogonality property $\left\langle\varphi_{1}(T) g_{1}(T) \mid \varphi_{2}(T) g_{2}(T)\right\rangle=0$. For $t \geq t_{\min },\left|g_{1}(t)\right\rangle$ represents the free outgoing wave packet for the spectator particle 1 , having an average momentum

$$
\begin{aligned}
\left\langle q_{1}\right\rangle_{\mathrm{el}}(t) & =\frac{\left\langle g_{1}(t)\left|q_{1}\right| g_{1}(t)\right\rangle}{\left\langle g_{1}(t) \mid g_{1}(t)\right\rangle} \\
& =\frac{\left\langle f_{1}(t)\left|\mathcal{S}_{\mathrm{el}}^{\dagger} q_{1} \mathcal{S}_{\mathrm{el}}\right| f_{1}(t)\right\rangle}{\left\langle g_{1}(t) \mid g_{1}(t)\right\rangle},
\end{aligned}
$$

and with its average relative separation from the bound pair being

$$
\begin{aligned}
\left\langle y_{1}\right\rangle_{\mathrm{el}}(t) & =\frac{\left\langle g_{1}(t)\left|y_{1}\right| g_{1}(t)\right\rangle}{\left\langle g_{1}(t) \mid g_{1}(t)\right\rangle} \\
& =\frac{\left\langle f_{1}(t)\left|\mathcal{S}_{\mathrm{el}}^{\dagger} y_{1} \mathcal{S}_{\mathrm{el}}\right| f_{1}(t)\right\rangle}{\left\langle g_{1}(t) \mid g_{1}(t)\right\rangle} .
\end{aligned}
$$

For the free time evolution of $\left|\Phi_{1}(t)\right\rangle$, the average of $y_{1}$ is computed as $\left\langle y_{1}\right\rangle_{\text {free }}(t)=\left\langle f_{1}(t)\left|y_{1}\right| f_{1}(t)\right\rangle$. Of course, the average momentum of the free wave packet should come out as $q_{0}$. For $t \geq t_{\min },\left\langle q_{1}\right\rangle_{\mathrm{el}}$ should also be constant, which, however, will be in general different than $q_{0}$. Since the coordinate-space representations $\left\langle y_{1} \mid u_{1 m}\right\rangle$ of the momentum-space basis functions can be computed analytically, and stored, computer time needed to calculate the average positions is minimal.

Table I gives $\left\langle y_{1}\right\rangle_{\mathrm{el}},\left\langle y_{1}\right\rangle_{\text {free }}$, and $\Delta y_{\mathrm{el}}$ at a number of sampling times $T$. Here, $\Delta y_{\mathrm{el}}$ is the position dispersion of the spectator wave packet $\left|g_{1}(t)\right\rangle$. Note that $\left\langle y_{1}\right\rangle_{\text {free }}(t)$ have been computed using the numerically propagated free wave packet, and differ only slightly from the theoretically expected values for $t \leq 4.0$. For example, at $T=4.0,\left\langle y_{1}\right\rangle_{\text {free }}^{\text {num }}=15.03 \mathrm{fm}$, whereas $\left\langle y_{1}\right\rangle_{\text {free }}^{\text {exact }}=15.00$ $\mathrm{fm}$. Of course, a much higher degree of accuracy can be achieved for the (separable) free-wave-packet propagation by using a finer discretization basis, but this would create a mismatch between numerical treatments of $\left|\Phi_{1}(t)\right\rangle$ and $\left|\Psi_{S}(t)\right\rangle .{ }^{21}$

Note also that the (numerical) average speeds associated with $\left|f_{1}(t)\right\rangle$ and $\left|g_{1}(t)\right\rangle$ increase from $6.0 \mathrm{fm}^{-1}$ at $t=2.5$ to about $6.1 \mathrm{fm}^{-1}$ at $t=5.5$, with the speed increase being more noticeable after $t \geq 4.0$. This is presumably due to the inability of the basis sets to represent the fast oscillations of the wave packets at large times. Nevertheless, the time dependence of $\left\langle y_{1}\right\rangle_{\mathrm{el}}$ for $2.5 \leq t \leq 4.0$ is consistent with that of a free outgoing spectator packet of average momentum $4.0 \mathrm{fm}^{-1}$ (average speed $6.0 \mathrm{fm}^{-1}$ ). 
Also, there is no indication of boundary reflection occurring. Had boundary reflection occurred, $\left\langle y_{1}\right\rangle_{\text {el }}$ would eventually have stopped growing linearly with $t$, and, at some stage, would have started to decrease.

Also shown in Table $\mathrm{I}$ is the average elastic $S$ matrix $\left\langle\mathcal{S}_{\text {el }}\right\rangle$, computed at different sampling times. Here, $\left\langle\mathcal{S}_{\text {el }}\right\rangle$ is the average of $\mathcal{S}_{\text {el }}$ over the momentum distribution of the initial wave packet, i.e.,

$$
\begin{aligned}
\left\langle\mathcal{S}_{\text {el }}\right\rangle & =\left\langle\Phi_{1 q_{0}}(T)\left|\mathcal{S}_{e l}\right| \Phi_{1 q_{0}}(T)\right\rangle=\left\langle\Phi_{1 q_{0}}(T) \mid \Psi_{S q_{0}}(T)\right\rangle \\
& =\left\langle f_{1 q_{0}}(T)\left|\hat{\mathcal{S}}_{\mathrm{el}}\right| f_{1 q_{0}}(t)\right\rangle=\left\langle f_{1 q_{0}}(T) \mid g_{1 q_{0}}(t)\right\rangle .
\end{aligned}
$$

The state-to-state elastic $S$ matrix elements $\mathcal{S}_{\text {el }}\left(E_{1 q}\right)$ computed from the same wave-packet solution via Eq. (21) are given in Table II for a range of $q$ values contained in the momentum distribution of the initial wave packet. Typically, $S$ matrix elements for initial states that have a probability density greater than about 0.01 in the initial wave packet can be extracted with reasonable accuracy. Note that the total breakup probabilities (computable as $\left.1-\left|\hat{\mathcal{S}}_{\text {el }}\right|^{2}\right)$ range from $\sim 22 \%$ at $q=3.0$ to $\sim 2 \%$ at $q=5.0$. Satisfactory results could be obtained up to $T=4.0$, which corresponds to a spectator separation of $15.2 \mathrm{fm}$. Taking the dispersion into account, the propagation of the same wave packet in coordinate space until $T=4.0$ would have required the cutoff value for $y$ to be at least $20 \mathrm{fm}$. The wave-packet results in Table II are typically accurate to second place after the decimal point. The least accurate ones are the values of $\operatorname{Im}\left(\mathcal{S}_{\mathrm{el}}\right)$ for $q=5.0 \mathrm{fm}^{-1}$, with an average error of about $15 \%$. The comparitively higher errors observed for $q=4.6-5.0$ can be traced back to the use of relatively small cutoff values $q_{\max }$ and $\kappa_{\max }$, and the use of a very small number of discretization points beyond $q=4.8$ and $\kappa=4.5$. That is, the high-momentum tail of the wave packet is not well approximated with the present basis, which can be remedied by using larger cutoff values and increasing the mesh points.

To show how boundary reflection manifests itself in momentum-space wave- packet propagation, we show in Table III the results of a calculation for the same ini- tial wave packet, but using a coarser discretization mesh with $\mathcal{M}_{1}=41, \mathcal{M}_{0}=29$, and $\mathcal{N}_{01}=13$. The propagation has been continued on purpose to larger times than necessary. The behavior of $\left\langle y_{1}\right\rangle$ and $\Delta y_{1}$ with time indicates clearly that boundary reflection starts around $t=6.0$, and $\left|g_{1}\right\rangle$ behaves like a free incoming wave packet after about $t=7.5$. Obviously, in this case the extraction of the $S$ matrix via Eq. (18) would be meaningful only prior to $t=6.0$.

A comparison of the $T$ dependences of $\left\langle y_{1}\right\rangle_{\text {free }}$ in Tables I and III indicates that there is considerable numerical scattering (or, numerical noise) in the propagation of even the free wave packet with the smaller basis. The deviations of $\left\langle y_{1}\right\rangle_{\text {free }}$ from theoretically expected values are quite significant, and the free wave packet seems to accelerate from $t=2.5$ to about $t=5.5$. Also, the wave-packet dispersions at the same sampling time are different in the two sets of calculations, with the smaller basis showing additional numerical spreading. The source of this noise is twofold: First, the actual initial wave packet used is not $f_{1 q_{0}}$ given in Eq. (2), but rather its approximate expansion (9). That is, the numerical initial conditions of the two sets of calculations are not quite equivalent, and the initial numerical wave packets have different dispersions to start with, although their initial average positions agree to four significant figures. In particular, the position dispersion of $f_{1 q_{0}}^{\text {num }}(0)$ is $1.44 \mathrm{fm}$ for $\mathcal{M}_{1}=59$, and $1.64 \mathrm{fm}$ for $\mathcal{M}_{1}=41$, whereas the exact dispersion of the analytical form $(2)$ is $\sqrt{2}(=w / \sqrt{2})$. Second, there is the numerical noise coming from the approximate evolution equation. Although the momentum-space wave packet retains its envelope, its frequency of oscillations will increase with time, as Eq. (4) indicates for a free wave packet. Especially difficult to represent in a basis will be the high-momentum components of the wave packet at large times. Therefore, a given momentum-discretization mesh will cease to be adequate after a certain time. Of course, boundary reflection and recurrence phenomena will show up if one insists upon continuing the propagation indefinitely.

For longer wave-packet propagation, such as that which would be needed with initial wave packets of low

TABLE III. $\left\langle y_{1}\right\rangle_{\mathrm{free}},\left\langle y_{1}\right\rangle_{\mathrm{el}}, \Delta y_{\mathrm{el}}$, and $\left\langle\mathcal{S}_{\mathrm{el}}\right\rangle$ as a function of the sampling time $T$. The wavepacket parameters are the same as in Table I, but expansion basis is smaller: $\mathcal{M}_{1}=41, \mathcal{M}_{0}=29$, and $\mathcal{N}_{01}=13$.

\begin{tabular}{cccccc}
\hline \hline$T$ & $\left\langle y_{1}\right\rangle_{\text {free }}$ & $\left\langle y_{1}\right\rangle_{\text {el }}$ & $\Delta y_{\text {el }}$ & $\operatorname{Re}\left\langle\mathcal{S}_{\text {el }}\right\rangle$ & $\operatorname{Im}\left\langle\mathcal{S}_{\text {el }}\right\rangle$ \\
\hline 3.00 & 9.09 & 9.25 & 2.38 & 0.949 & 0.139 \\
4.00 & 15.32 & 15.52 & 4.19 & 0.940 & 0.140 \\
5.00 & 22.24 & 22.46 & 7.45 & 0.923 & 0.147 \\
6.00 & 29.34 & 29.37 & 10.08 & 0.910 & 0.154 \\
6.50 & 31.16 & 30.96 & 10.35 & 0.911 & 0.155 \\
7.00 & 30.81 & 30.39 & 10.29 & 0.919 & 0.154 \\
7.50 & 28.62 & 28.13 & 10.02 & 0.922 & 0.150 \\
8.00 & 25.41 & 25.04 & 9.44 & 0.916 & 0.146 \\
Exact & & & & 0.953 & 0.145 \\
\hline \hline
\end{tabular}


average momentum, a finer discretization of momentum space is needed if serious numerical scattering and boundary reflection are to be avoided before the wave packet emerges from the interaction region. (The coordinatespace counterpart of this requirement is the need for a larger computational domain.) Note that some numerical noise can be tolerated if the $S$ matrix is extracted by a comparison of the numerical wave packet with the numerical free wave packet. ${ }^{21}$ In other words, as long as boundary reflection is avoided, even a relatively crude wave-packet calculation can provide meaningful $S$ matrix information (especially the average $S$ matrix, as Table III indicates).

\section{CONCLUSIONS}

As the results of Sec. VI indicate, the proposed method is quite efficient in describing the wave-packet dynamics of a reactive system. Considering the success of the coupled-reaction-channel (CRC) methods ${ }^{15}$ in timeindependent descriptions of rearrangement collisions, this is not surprising. The present method involves an extension of the conventional CRC ansatz by augmenting it with an explicit breakup term. Although we used the extended ansatz in the time-dependent context, it could also be used within a stationary description as well. The latter would essentially correspond to a computational implementation ${ }^{24}$ of the Chandler-Gibson theory.

Although we have not done so in this paper, the stateto-state breakup $S$ matrix elements can also be extracted from the wave packet. Denoting the distinguishable breakup $S$ operator with $S_{01}$, and introducing the symmetrized breakup operator $\mathcal{S}_{\mathrm{bp}} \equiv\left(I+P_{123}+P_{132}\right) S_{01}$. we have, in the context of the present test problem, $\left|\Psi_{\mathrm{bp}}(T)\right\rangle=\sqrt{3} \mathcal{S}_{\mathrm{bp}}\left|\Phi_{1 n_{0} q_{0}}(T)\right\rangle$, for $T \geq t_{\min }$. Numerical implementation of this scheme is currently in progress.

Other than the expansion ansatz adopted, two other aspects of the present calculations deserve comment: (i) The use of the numerically propagated free wave packet in extracting the sharp-energy $S$ matrices via Eq. (15), and (ii) the propagation of the wave packet in momentum space. Concerning the first point, we note that most wave-packet methods implicitly involve the replacement of the Hamiltonian (and the corresponding evolution operator) by a finite-rank approximation. Since the $S$ matrix basically involves a comparison of the full Hamiltonian with channel Hamiltonians, it is imperative that they be treated to the same level of approximation. Empirically, we find that this allows for cancellation of errors arising from the treatments of the kinetic-energy operators. Since the handling of the kinetic-energy operators in coordinate-space calculations presents somewhat of a bottleneck, this procedure might also prove useful in that context.

The advantages of the momentum space lie in the nonmoving and nonspreading nature of the momentum-space wave packets, and the locality of the kinetic-energy operators. Thus, the computational momentum-space domain needed is basically determined by the effective momentum support of the wave packet. On the other hand, the numerical treatment of the kinetic-energy operators does not require excessively large bases (or fine discretization meshes). The fineness of the discretization is determined to a large extent by the maximum time of propagation required by the collision process under consideration. It is remarkable that the relatively small discretization basis used in the present calculations is capable of describing the wave-packet propagation up to final relative separations of about $15 \mathrm{fm}$. A corresponding calculation in coordinate space would have required a computational cutoff value of $\sim 20 \mathrm{fm}$ for $y$. Hence, unless a moving mesh or absorptive boundaries were employed, a larger number of coordinate mesh points would probably have been needed than that used in the present momentumspace discretization.

However, potentials in momentum space become integral operators, which upon discretization yield full matrices, whereas most coordinate-space discretizations result in banded matrices. Since most time-propagation algorithms can be arranged as repeated matrix-vector multiplications, this is a serious disadvantage. Calculation of the matrix elements of local potentials in a momentum-space basis can be seen as another disadvantage. However, this is not a serious problem, because necessary integrals can in fact be carried out in coordinate space since the piecewise interpolation functions (of momenta) can be analytically transformed into the coordinate space. Note that coordinate-space wave packets also have to be transformed to the momentum space for the final analysis. In addition, in coordinate-space methods employing the fast-Fourier-transform method to treat the kinetic-energy operators, the wave packet is transformed back and forth between coordinate and momentum spaces many times, as required by the particular time-propagation scheme adopted.

Overall, relative computational efficiencies of the coordinate- and momentum-space wave-packet methods would hinge upon whether the possible reduction of matrix dimensions in momentum space is enough to offset the greater computational cost of repeated matrix-vector multiplications involving full matrices.

\footnotetext{
${ }^{1}$ For recent large-scale TDWP calculations of moleculesurface and nonreactive atom-diatom collisions, see R.C. Mowrey, Y. Sun, and D.J. Kouri, J. Chem. Phys. 91, 6519 (1989); Y. Sun, R.C. Mowrey, and D.J. Kouri, ibid. 87, 339 (1987), and references cited therein.
}

${ }^{2}$ For a review of pre-1987 TDWP methods and calculations in the context of chemical physics, see the review article by V. Mohan and N. Sathamurty, Comput. Phys. Rep. 7, 214 (1988).

${ }^{3}$ X.P. Jiang, R. Heather, and H. Metiu, J. Chem. Phys. 90, 
2555 (1989), and references cited therein.

${ }^{4}$ D.J. Kouri and R.C. Mowrey, J. Chem. Phys. 86, 2087 (1987). For a different method based on integral-equation formulation of time-dependent scattering theory, see J. Holtz and W. Glockle, Phys. Rev. C 37, 1390 (1988).

${ }^{5}$ For TDWP calculations of collinear atom-diatom reactions, see E.A. McCullough and R.E. Wyatt, J. Chem. Phys. 54, 3578 (1971); K.C. Kulander, ibid. 69, 5064 (1978); P.M. Agrawal and L.M. Raff, ibid. 74, 5076 (1981); R. Kosloff and D. Kosloff, ibid. 79, 1823 (1983); C. Leforestier, Chem. Phys. 87, 241 (1984); Z.H. Zhang and D.J. Kouri, Phys. Rev. A 34, 2687 (1986); D. Neuhauser and M. Baer, J. Chem. Phys. 91, 4651 (1990).

${ }^{6}$ D. Neuhauser, M. Baer, R.S. Judson, and D.J. Kouri, J. Chem. Phys. 90, 5882 (1989).

${ }^{7}$ Z.C. Kuruoglu and F.S. Levin, Phys. Rev. Lett. 64, 1701 (1990).

${ }^{8}$ For a discussion of breakup boundary conditions, see, e.g., S.P. Merkuriev, C. Gignoux, and A. Laverne, Ann. Phys. (N.Y.) 99, 30 (1976); W. Glockle, Z. Phys. 271, 31 (1974).

${ }^{9}$ Z.C. Kuruoglu and F.S. Levin, Phys. Rev. C 36, 49 (1987).

${ }^{10} \mathrm{C}$. Chandler and A. Gibson, J. Math. Phys. 14, 2336 (1977).

${ }^{11}$ P.M. Prenter, Splines and Variational Methods (Wiley, New York, 1975); R. Wait and A.R. Mitchell, Finite Element Analysis and Applications (Wiley, New York,1985); C.A.J. Fletcher, Computational Galerkin Methods (Springer, New York, 1984).

${ }^{12}$ H. Tal-Ezer and R. Kosloff, J. Chem. Phys. 81, 3967 (1984); M.D. Feit, J.A. Fleck, Jr and A. Steiger, J. Comp. Phys. 47, 412(1983); T.J. Park and J.C. Light, J. Chem. Phys. 85, 5870 (1986).

${ }^{13}$ A. Askar and A.S. Cakmak, J. Chem. Phys. 68, 2794 (1978).

${ }^{14}$ Z.C. Kuruoglu and F.S. Levin, Phys. Rev. Lett. 48, 899 (1982), and Ann. Phys. (NY) 163, 120 (1985).

${ }^{15}$ For a review of the CRC method in the context of nuclear reactions, see Y.C. Tang, M. LeMere, and D.R. Thompson, Phys. Rep. 47, 167 (1978). For the use of the CRC method for chemical reactions, see W.H. Miller, J. Chem. Phys. 50, 407 (1969), D.W. Schwenke, D.G. Truhlar, and D.J.
Kouri, ibid. 86, 2772 (1987), J.Z.H. Zhang, D.J. Kouri, K. Haug, D.W. Schwenke, Y. Shima, and D.G. Truhlar, ibid. 88, 2492 (1988), D.W. Schwenke, K. Haug, M. Zhao, D.G. Truhlar, Y. Sun, J.Z.H. Zhang, and D.J. Kouri, J. Phys. Chem. 92, 3202 (1988), and J.Z.H. Zhang and W.H. Miller, J. Chem. Phys. 88, 4454 (1988); 88, 4459 (1988).

${ }^{16}$ A. Ben-Israel and T.N.E. Greville, Generalized Inverses: Theory and Applications (Wiley, New York, 1974). The use of generalized inverses to handle the overcompleteness problem of the CRC method is discussed in Gy. Bencze, C. Chandler, and A.G. Gibson, Nucl. Phys. A 390, 461 (1982); M.C. Birse and E.F. Redish, Nucl. Phys. A 406, 149 (1983).

${ }^{17}$ See, e.g., W. Glockle, The Quantum Mechanical Few Body Problem (Springer, Berlin, 1983).

${ }^{18} \mathrm{~A}$ good discussion of, and references for, various hyperspherical coordinates are given by R.T. Pack and G.A. Parker, J. Chem. Phys. 87, 3888 (1987). For examples of hyperspherical momentum representations, see S. Boukraa and J.L. Basdevant, J. Math. Phys. 30, 1060 (1989); R.I. Dzhibuti and Sh.M. Tsiklauri, Yad. Fiz. [Sov. J. Nucl. Phys. 41, 554 (1985)].

${ }^{19}$ J.R. Taylor, Scattering Theory (Wiley, New York, 1972).

${ }^{20}$ The sharp-energy states making up the wave packet do not necessarily evolve independently during numerical propagation, even though the average energy of the packet might be conserved within acceptable limits. That is, it is much easier in numerical calculations to satisfy the conservation of average energy than to satisfy the state-to-state energy conservation.

${ }^{21}$ This point is planned to be discussed in greater detail and with numerical examples in a forthcoming paper.

${ }^{22}$ M.L. Goldberger and K.M. Watson, Collision Theory (Wiley, New York, 1964), p. 139.

${ }^{23}$ Z.C. Kuruoglu and D.A. Micha, J. Chem. Phys. 80, 4262 (1984).

${ }^{24}$ C. Chandler and A.G. Gibson, J. Math. Phys. 30, 1533 (1989). A time-independent approach similar in spirit to the Chandler-Gibson theory has recently been announced by D.J. Kouri and D.K. Hoffman (private communication). 